\title{
Grounding Reichenbach's Pragmatic Vindication of Induction
}

\author{
Michael J. Shaffer \\ Department of Philosophy \\ St. Cloud State University
}

\begin{abstract}
This paper has three interdependent aims. The first is to make Reichenbach's views on induction and probabilities clearer, especially as they pertain to his pragmatic justification of induction. The second aim is to show how his view of pragmatic justification arises out of his commitment to extensional empiricism and moots the possibility of a non-pragmatic justification of induction. Finally, and most importantly, a formal decision-theoretic account of Reichenbach's pragmatic justification is offered in terms both of the minimax principle and of the dominance principle.
\end{abstract}

\section{Introduction}

Hans Reichenbach's pragmatic treatment of the problem of induction (presented and developed in his 1949a, 1932/1949b and 1949c) was, and still is, of great interest. However, various influential commentators have dismissed it as a pseudo-solution and/or regarded it as problematically obscure. ${ }^{1}$ This is, in large part, due to the difficulty in understanding exactly what Reichenbach's solution is supposed to amount to, especially as it appears to offer no response to the inductive skeptic. As Laurence BonJour claims,

...the significance of Reichenbach's pragmatic justification remains obscure. As he himself insists, that justification still yields no reason at all for thinking that inductive conclusions, or any of the myriad further beliefs which are epistemically dependent on them, are to any degree likely to be true. The sort of justification in question is thus not epistemic justification, as that concept was construed above; to show that beliefs are justified in this alternative way does not answer, or even purport to answer, the basic skeptical worry about induction, and is indeed quite compatible with the deepest degree of skepticism. It is thus hard to see why it should be regarded as any sort of solution to the classical problem of induction (BonJour 1986, p. 99).

1 See, e.g., Skyrms (1966), Salmon (1966), BonJour (1986), BonJour (1992), BonJour (1998), Rosenkrantz (1981), and Kelly (1991). 
This sort of dismissal of Reichenbach's views on induction surely depends to some degree on his adherence to the controversial frequency interpretation of the concept of probability. ${ }^{2}$ This is plausible to assume in light of the wide-spread popularity of subjectivism about probability that has dominated probability theory subsequent to Reichenbach's work and this aspect of Reichenbach's approach to induction has been treated at length in a variety of other places. ${ }^{3}$ Another aspect of his vindication that likely fuels the charge of obscurity is the failure of these critics to pay more attention to Reichenbach's commitment to a purely extensional metaphysics. Finally, Reichenbach's attempt to rationally ground induction on the basis of purely pragmatic considerations is also likely to have, at least in part, given rise to the charges of obscurity and failure. ${ }^{4}$ This last point is especially important because Reichenbach is rather cavalier and informal in his assertion of the claim that following the inductive rule is the best thing to do from a pragmatic perspective.

First, it will be shown here that what Reichenbach does in his later work on induction is to establish an important epistemic limitation of extensional empiricism and that there really is nothing especially obscure about Reichenbach's thoughts on induction in this respect. He was simply working out the limits of extensional empiricism with respect to inductive inference. In fact, Reichenbach conveys this very point to Russell in an important letter in $1949 .{ }^{5}$ It will be shown that these aspects of Reichenbach's position lead directly to his assertion that the only manner in which the inductive rule can be justified in the primitive state of knowledge prior to making sequences of inductive inferences is pragmatic in nature. Finally, and more constructively, it will be shown here that there are perfectly coherent, formal and pragmatic justifications of Reichenbach's inductive rule in the primitive state of knowledge in the forms of the maximin rule for decision making and the dominance principle. With this account of the pragmatic justification of induction in hand, we will see

\footnotetext{
${ }^{2}$ Hájek (1997, 2009).

3 See, e.g., Skyrms (1966), Salmon (1963), Salmon (1966), Galvaotti (2011) and Teng and Kyburg (2001).

${ }^{4}$ The sense of pragmatics used here is just the idea that there are justifications that are non-epistemic (i.e. not related to truth, approximate truth or probability) and which are based on some more or less well-understood notion of instrumental success or utility.

5 The letter is a response to Russell's criticisms of Reichenbach's approach to the problem of induction as presented in Russell's Our Knowledge of the External World. This approach stands in sharp contrast to Reichenbach's early work which resembles Russell's approach in holding that the principle that grounds induction is synthetic a priori. See Eberhardt (2011) for discussion of Reichenbach's early views. He explicitly rejects this view in his later work and tells Russell in a 1949 letter that "Induction does not require an intensional logic" (1949d, p. 410).
} 
that Reichenbach's justification of induction can be given a principled ground.

\subsection{Overview}

Let us begin by recalling that Reichenbach's pragmatic justification of induction is based on the following (reconstructed) line of argumentation (i.e. the basic Reichenbach argument):

$\mathrm{P} 1$ : Either nature is uniform or it is not.

P2: If nature is uniform, then scientific induction will be successful.

P3: If nature is not uniform, then no method will be successful.

$\therefore$ If any method of induction will be successful, then scientific induction will be successful. $^{6}$

But, according to Reichenbach and echoing Hume, we cannot know whether nature is uniform or not, because it is neither a matter that can be settled a priori nor is it a matter that we can non-circularly establish a posteriori. So, as Reichenbach sees it, although we know that if any method is successful, then scientific induction will be successful, we cannot know that any method really is successful. The gist of his attempt to justify inductive practice then comes from the idea that while we do not know that any method will actually be successful, we also do not know that no method will be successful. Given this result and the fact that scientific induction can be shown to be an optimal method (in this important sense of "optimality"), we ought to accept induction as being justified, at least pragmatically speaking. As we shall see, what is at the heart of this view is Reichenbach's metaphysical commitment to a form of extensional empiricism that tolerates only the existence of particulars.

In any case, as Salmon correctly pointed out in his 1966, the Reichenbach argument depends on a false dichotomy. The uniformity of nature is, of course, not an all or nothing matter. We can, of course imagine possible worlds that contain only individuals with degrees of uniformity that vary radically. So, the uniformity of nature seems to be a matter of degree, and it is at least plausible to believe that there is a measure of the uniformity of extensional worlds. If this turns out to be viable, given the space of possible worlds $U$, we could define a measure $m(x)$ on $U$ such that $m(x)$ maps the elements of $U$ into the continuous open interval $[0,1]$

\footnotetext{
${ }^{6}$ This presentation of a simplified version of Reichenbach's main argument is taken from Skyrms (1966). It is important to note at this juncture that the various criticisms of Reichenbach's views, other than Russell's, will (for the most part) be ignored here. To address all of those criticisms would require too much space, and the point of this paper is more historical in any case.
} 
representing the uniformity of that extensional world. This suggests that Reichenbach's attempt to justify induction needs to be retooled in order to accommodate a concept of world-uniformity that admits of continuous degrees. When this is done we can usefully reformulate the basic Reichenbach argument as follows. Consider our world $w_{\mathrm{a}}$ (the actual world), where $w_{\mathrm{a}} \in U$, with a fixed but unknown measure of uniformity, the set of all inductive methods $y_{,}{ }^{7}$ where $y_{\mathrm{i}} \in \mathcal{Y}$ and such that each inductive method has a probability of arriving at a true conclusion in its domain of application, a function $f\left(m\left(w_{\mathrm{n}}\right), y_{\mathrm{n}}\right)$ that maps worlds with degrees of worlduniformity and inductive methods into the space of probabilities, ${ }^{8}$ and a constant $\lambda$ that represents the chance probability of an inductive method succeeding at a world. ${ }^{9}$ If we understand $\varepsilon$ as the degree of worlduniformity required for any inductive rule to be reliable with a reliability greater than chance, i.e. greater than $\lambda$, then the more sophisticated Reichenbach argument can be stated as follows:

P1': If the probability that $m\left(w_{\mathrm{a}}\right)=1$ is 1 , then scientific induction will be successful.

P2': If it is probable that $1>m\left(w_{\mathrm{a}}\right)>\varepsilon$ with probability less than 1 but greater than $\lambda$, then scientific induction will be successful with probability $p$, where $p>\lambda<1$.

P3': If it is probable that $\varepsilon>m\left(w_{\mathrm{a}}\right)>0$ with probability greater than 0 but less than $\lambda$, then scientific induction will be successful with probability $p$, where $p<\lambda<0$.

P4': If the probability that $m\left(w_{\mathrm{a}}\right)=0$ is 1 , then no inductive method will be successful.

$\therefore$ If any inductive method will be successful, scientific induction will be successful.

It should be noted that Reichenbach's conclusion still holds in this case, and we will consider the significance of this conclusion in what follows.

7 Inductive methods are, simply, rules for accepting conclusions concerning unobserved cases based on observed cases.

${ }^{8}$ The function $f\left(m\left(w_{\mathrm{n}}\right), y_{\mathrm{n}}\right)$ seems, intuitively, to be a natural sort of function, as degrees of world-uniformity seem to be closely related to the probability with which a method produces true conclusions. What $f\left(m\left(w_{\mathrm{n}}\right), y_{\mathrm{n}}\right)$ is supposed to yield is a probabilistic measure of the general reliability of a given method at a world with a given measure of uniformity, and, as we shall see in section 4, what this function really represents is the set of worlds where an inductive method with a well-defined probability of arriving at the correct value of a stable frequency will actually produce the correct values.

${ }^{9}$ In other words, $\lambda$ represents the threshold at which methods are no better at producing true conclusions than randomly selecting conclusions from the set of all statements of a given language $£$, and, as we shall see, a method that performs at a success rate no better than chance is no method at all. However, the general successfulness of an inductive method will turn out to be a more complex matter involving two aspects. The first concerns the reliability of the procedure in its domain, and the second concerns whether there exist elements of that domain at a world. 
However, before we proceed to do so, it will be instructive to reconstruct Reichenbach's treatment of induction in much greater detail in order to see just what it amounts to and what it implies about inductive inference.

\section{Reichenbach's Conception of Scientific Induction}

The primary motivation that drove Reichenbach to propose his pragmatic justification of induction concerns a central feature of the frequency interpretation of the probability calculus. Familiarity with the details of the probability calculus will be assumed here, and with the fact that it is compatible with at least several interpretations. The axioms of the probability calculus are, of course, as follows:

(A.1) $\mathrm{P}(a) \geq 0$ for all $a$ in the domain of $\mathrm{P}(\cdot)$.

(A.2) $\mathrm{P}(t)=1$ if $t$ is a tautology.

(A.3) $\mathrm{P}(a \vee b)=\mathrm{P}(a)+\mathrm{P}(b)$ if $a$ and $b$ and $a \vee b$ are all in the domain of $\mathrm{P}(\cdot)$, and $a$ and $b$ are mutually exclusive.

Recall that on Reichenbach's frequency interpretation of probabilities such quantities are to be construed as tautological consequences of the probability calculus. ${ }^{10}$ More importantly, probabilities are to be regarded as measures of the limit of the relative frequency with which one contingent property is associated with another in an infinite sequence. More formally, the relative frequency of a pair of properties in a sequence is to be defined as follows:

$$
F^{n}(\mathrm{~A}, \mathrm{~B})=N^{n}(\mathrm{~A}, \mathrm{~B}) / N^{n}(\mathrm{~A})
$$

Here $F^{n}(\mathrm{~A}, \mathrm{~B})$ is the frequency of associated As and Bs in a sequence of length $n$. Given this conception of relative frequency we can then define the concept of probability as follows:

$$
\begin{aligned}
& P(\mathrm{~A}, \mathrm{~B})=\lim F^{n}(\mathrm{~A}, \mathrm{~B}) .{ }^{11} \\
& n \rightarrow \infty
\end{aligned}
$$

Having introduced this notion of probability Reichenbach then proposes the rule of induction that states,

If an initial section of $\mathrm{n}$ elements of a sequence $x_{\mathrm{i}}$ is given, resulting in the frequency $f^{n}$, and if, furthermore, nothing is known about the probability of the second level for the occurrence of a certain limit $p$, we posit that the frequency $f^{i}(i>n)$ will approach a limit $p$ within $f^{n} \pm \delta$ when the sequence is continued (Reichenbach, 1949c, p. 47).

However, these definitions give rise to some very difficult but well-known problems concerning the existence of infinite sequences and the existence

\footnotetext{
${ }^{10}$ See Reichenbach (1949b) and Weatherford (1982), chapter 4.

${ }^{11}$ See Reichenbach (1949c) for details concerning how this derivation is carried out.
} 
of such convergent limits. ${ }^{12}$ Reichenbach assumes that we are only ever aware of sequences that "... are not intensionally given, but are presented to us only by enumeration of their elements, i.e. are extensionally given (Reichenbach, 1949a, p. 309)" and that any such sequence of observed associations will be finite. Upon considering further extensional enumeration of the elements of a given observed sequence, we find that such extended sequences are, in point of fact, compatible with any value of the limit frequency. If this is so, we might ask why we are entitled in any way to assume that the frequency of such an association in even very long sequences of observed associations in a population will justify our assertion that that frequency will not diverge in further extensive enumerations of that sequence. Unless one is prepared to reject extensional empiricism this conclusion seems inescapable. Adopting intensionalism and accepting the existence of the relevant sort of a priori knowledge, like Russell ultimately did (1912 and 1948), permits induction to be grounded in a robust essentialism and a form of rationalism that allows for synthetic a priori knowledge of universals. But, although Reichenbach himself endorsed such a view in his early thinking about this problem in his dissertation, in his later work he staunchly opposed this sort of view and the idea of a priori knowledge, claiming that, "The idea that there is such a thing as a 'rational belief' is the root of all evil in the theory of knowledge and is nothing but a remnant from rationalistic philosophies (Reichenbach, 1949d)."

In any case, Reichenbach saw that his understanding of this problem in terms of frequentism was just the classical problem of induction in a somewhat new guise, and he showed two things. First, he showed that, by definition, if such a limit exists, then the procedure of scientific induction will be successful, and, second, that scientific induction is at least as good as any other method in discovering what is really the case concerning the frequency of an association in a sequence. Reichenbach explains,

Let us assume for the moment that there is a limit towards which the sequence converges, then there must be an $n$ from which on our posit [the rule of induction] leads to the correct result; this follows from the definition of the limit, which requires that there be an $n$ from which on the frequency remains within a given interval $\delta$. If we were to adopt, on the contrary, the principle of always positing a limit outside $f^{n} \pm \delta$ when a frequency $f^{n}$ has been observed, such a procedure would certainly lead us to a false result from a certain $n$ on. This does not mean that there could not be other principles which like the first [the rule of induction] would lead to the correct limit. But we can make the following statement about these principles: even if they determine the posit outside $f^{n} \pm \delta$ for a smaller $n$, they must,

12 Sequences with convergent limiting frequencies are just those sequences that settle into stable frequencies in the limit. 
from a certain $n$ on, determine the posit within $f^{n} \pm \delta$. All other principles of positing must converge asymptotically with the first [the rule of induction] (Reichenbach, 1949b, p. 316).

What he showed was that if a limit exists for a sequence, then by repeated application the rule of induction will lead to the value of that limit to any desired degree of approximation in a finite number of applications, and that all other methods will asymptotically converge with the results of the rule of induction. So, in spite of the fact that we cannot know that the limiting frequencies of sequences exist, we might as well simply accept the rule of induction because it is the best method of all methods. All methods are, in a sense, parasitic on the rule of induction. Again, this pragmatic answer to the problem of induction arose directly out of Reichenbach's recognition that, in point of fact, we cannot know that such limits exist in our world. We cannot know whether such convergent limits exist based on the empirical observation of associations in finite, extensionally given, sequences. So, we are stuck in the situation that either no method at all works, or induction is the best of all methods. In terms of the sophisticated Reichanbach argument this can be expressed as follows. If worlds are extensional and there is no a priori knowledge of the regularity of the world, then we cannot know the real value of $m\left(w_{\mathrm{a}}\right)$. Nevertheless, it will be true that if $1 \geq m\left(w_{\mathrm{a}}\right)>\varepsilon$, then scientific induction will be successful. If this is not the case, then no method will be successful.

\section{Posits and Pragmatic Justifications}

Even so, Reichenbach tells us that we can treat the existence of such limits of relative frequencies as posits, where posits are not to be treated as beliefs in the normal sense, but rather as a kind of wager concerning what would be most advantageous to us. Reichenbach explains that,

It is evidently the concept of posit which we have to employ for an explanation of this method. If in the finite section given we have observed a certain frequency

$f^{\mathrm{n}}$, we posit that sequence, on further continuation, will converge towards the limit $f$

${ }^{\mathrm{n}}$ (more precisely: within the interval $f^{\mathrm{n}} \pm \delta$ ). We posit this; we do not say that it is true, we only posit it in the same sense as the gambler lays a wager on the horse which he believes to be fastest. We perform an action which appears to us the most favorable one, without knowing anything about the success of this individual action (Reichenbach, 1949b, p. 315).

Furthermore, as all other rules are parasitic on the rule of induction, it is only natural to lay our wager on that rule. We are wagering that $1 \geq m\left(w_{\mathrm{a}}\right)>\varepsilon$. So, the sort of justification his argument provides is clearly a matter of pragmatics. It is, as Feigl claimed, a justification actionis. ${ }^{13}$ But,

\footnotetext{
${ }^{13}$ See Feigl (1963).
} 
in any case, the kind of wager involved in positing the existence of convergent limits in infinite sequences is not the typical kind of wager that a gambler makes. Normally, a gambler at least knows the odds with which he is confronted and so can make an informed decision about what outcome to bet on (i.e. which is the best bet), but in the case of the limits of infinite sequences we are making the posit that the limit converges to $f^{\mathrm{n}}$ blindly; i.e. we are making this posit when we do not know the odds, and so we do not know if it is the best posit.

Reichenbach claims that in such cases we are making what he calls an approximative posit concerning the existence of such limits. We are blindly wagering that $1 \geq m\left(w_{\mathrm{a}}\right)>\varepsilon$. As we have seen, Reichenbach shows that if we are right about the existence of such a limit (if this blind wager is correct), then induction will be successful, and if any other method is successful, then scientific induction will be successful. If we are wrong about the existence of such a limit (if this blind wager is not correct), then if any other method is successful, then scientific induction will be successful in this more restricted sense. Therefore, scientific induction is at least optimal in this specific sense. However, as BonJour notes in the passage quoted in section 1, this by no means shows that induction is justified in the traditional sense, and Reichenbach's view is apparently compatible with radical skepticism concerning the probity of induction. It may simply be false that $1 \geq m\left(w_{\mathrm{a}}\right)>\varepsilon$ and, given extensional empiricism, we cannot know whether this claim is true or false. So, as far as we know, the method of induction might well be the best of a bad lot. Nonetheless, Reichenbach argues that there is a sense in which his argument vindicates induction if one is committed to extensional empiricism. It does show that if any method works, then induction works. We do not know that the rule is unreliable, but we know that it is the best method if any method is reliable. So, why not commit ourselves to the use of scientific induction? Of course, this may not be a satisfactory justification for someone who has sympathies with BonJour's inductive skeptic, but it is clearly to our practical advantage if scientific induction turns out to be reliable. As Reichenbach sees it, the alternative is to give up on science altogether. More importantly for the purposes of this discussion, what this result really establishes is that given extensional empiricism induction can only be pragmatically justified in the sense of Reichenbach's vindication. But how? Reichenbach is not terribly clear on this point, but this lacuna can be fixed easily by appeal to the decision-theoretic maximin rule.

\section{The Maximin Justification of Reichenbach's Rule}

After presenting his account of the optimality of the inductive rule, Reichenbach discusses the sense in which using this rule can be justified in 
light of the problems associated with our ignorance of the existence of the convergent limit sequences that are required for the success of induction. As we have already seen, he says of the inductive rule that,

We posit this; we do not say that it is true, we only posit it in the same sense as the gambler lays a wager on the horse which he believes to be fastest. We perform an action which appears to us the most favorable one, without knowing anything about the success of this individual action (Reichenbach, 1949b, p. 315).

Elsewhere he also says:

Inductive positing in the sense of a trial-and-error method is justified so long as it is not known that the attempt is hopeless, that there is no limit of the frequency. Should we have no success, the positing was useless; but why not take our chance?

The phrase "take our chance" is not meant to state that there is a certain probability of success; it means only that there is a possibility of success in the sense that there is no proof that success is excluded (Reichenbach, 1949c, p. 49).

Finally, he explains to Russell in his 1949 letter that,

I have shown that blind posits are justified as a means to an end, and that no kind of belief in their truth is required. This I regard as an essential merit of my theory: I have shown that there are other reasons to make assertions than reasons based on belief (Reichenbach, 1949d, p. 407).

So, these remarks show that Reichenbach is clearly asserting that there are alternative justifications that have to do with non-epistemic pragmatic considerations and we know only that the probability that there are limit frequencies is non-zero. But, he also tells us in his 1949 letter that,

...the inductive conclusion can be called probable only when many other inductions have already been made, which tells us something about the second level probabilities. I speak here of the stage of advanced knowledge. In primitive knowledge, i.e. before any inductions were made, the inductive conclusion is not probable (Reichenbach, 1949d, p. 409).

So, in point of fact, Reichenbach believes that it is only when we have begun to make inductive inferences that we can even claim that the conclusions of inductive inferences are probable, and this is based on the success of making such inductions. Thus, the second-order probability of the inductive rule itself is very low in the state of primitive knowledge, for we have not therein yet made first-order inductions sufficient to gauge the second-order probability of induction. Prior to this state of advanced knowledge, inductive conclusions are not probable and can only be justified pragmatically in the sense of blind positing. Notice that this will be true for any alternative inductive rule as well. So, the rationality of induction writ large and independent of the selection of one of the possible reliable inductive rules depends on their being a vindication of the inductive rule in this primitive state. Even if there are other rules that are superior to Reichenbach's inductive rule in terms of how often they get correct results, 
this can only be determined in the state of advanced knowledge. All of this then suggests that Reichenbach's rather vague appeal to pragmatic justification can be made much more coherent by appealing to some particular resources in modern decision theory and focusing on justifying induction in the primitive state of knowledge.

In decision situations where probabilities cannot be meaningfully assigned to outcomes (other than that they are non-zero) advice about what to do is wholly a function of utilities, because no expected utilities can be calculated for such cases. There are then two ways one might provide such a pragmatic justification for adopting the inductive rule. First, in such cases and where the potential losses are great Wald's maximin rule usefully applies:

(MR) maximize the minimum utility outcome. ${ }^{14}$

Reichenbach's approximative posit fits this bill perfectly. Much is at stake here. If scientific induction does not work, then our inductive inferences cannot possibly succeed and we would have to give up on science. Moreover, as Reichenbach admits, we have no idea of the probabilities on which this wager is based. So, where $\mathrm{R}$ is ' $1 \geq m\left(w_{\mathrm{a}}\right)>\varepsilon$ ', $\mathrm{I}$ is 'employ scientific induction', $\mathrm{S}$ is 'assured success in inductive reasoning in the long run', $P$ is 'possible success in the long run', Reichenbach's wager can be fruitfully understood as follows: ${ }^{15}$

\begin{tabular}{|c|c|c|}
\hline & $\mathrm{R}$ & $\neg \mathrm{R}$ \\
\hline I & $\mathrm{S}$ & $\neg \mathrm{S}$ \\
\hline$\square \mathrm{I}$ & $\mathrm{P}$ & $\neg \mathrm{S}$ \\
\hline
\end{tabular}

${ }^{14}$ See Wald $(1939,1945)$ and Resnik (1987, pp. 26-27).

${ }^{15}$ Since we are only considering the primitive state of knowledge here, we need not be concerned with the claim that there are alternative rules of induction that are superior to Reichenach's preferred rule in our table. We can simply see that whatever rule in particular we adopt as the second-order guide to inductive inference, Reichenbach's considerations show us that the only kind of rational justification for any inductive rule (no matter how efficient) in the primitive state is a blind posit of this pragmatic sort. We could, in effect, run the very same argument substituting for I any such second-order rule. So, the upshot of Reichenbach's considerations really should be that the only justification for induction in the primitive state of knowledge is pragmatic. 
Here we also have the following relative ordering of utilities: $\mathrm{V}(\mathrm{S})>\mathrm{V}(\mathrm{P})>$ $\mathrm{V}(\neg \mathrm{S})$, and this is all the application of the maximin rule requires. We do not need to know ordinal utilities to apply MR. MR tells us to look at the worst outcomes of the acts given the relevant states of the world. For $\neg \mathrm{I}$ we get $\neg \mathrm{S}$, for I we also get $\neg \mathrm{S}$. These values are equal. By the lexical MR we then look at the next lowest outcome(s) of $\neg$ I and I respectively. In the case of I we get $\mathrm{S}$, and for $\neg \mathrm{I}$ we get P. So, the MR tells us that it is maximally rational to do I even in the primitive state of knowledge where inductive conclusions are not probable and where the second-order probability that the inductive rule is correct is also very low.

The second way one might flesh out Reichenbach's solution is in terms of a simple dominance argument based on the dominance principle. Again, where we have ignorance of the relevant probabilities we should look at the utilities of the outcomes and apply a principle of pragmatic rationality to decide what to do. The dominance principle is just such a rule. The familiar notion here is that an act $\mathrm{A}$ dominates an act $\mathrm{B}$ if for every outcome the utility of $\mathrm{A}$ is equal to or greater than the utility of $\mathrm{B}$ and for at least one outcome the utility of $\mathrm{A}$ is greater than that of $\mathrm{B}$. The dominance principle is then this:

(DOM) opt for dominant acts.

Referring back to our decision table we can see that I dominates $\neg$ I even in the primitive state of knowledge where inductive conclusions and the second-order probability that induction is correct is very low. Nevertheless, DOM tells us that it is pragmatically rational to opt for I. Thus, Rerichenbach's solution can be pragmatically vindicated, but in a principled rather than intuitive manner. It is pragmatically rational to use scientific induction and there is nothing at all obscure about why this is so. It is pragmatically justified in light of the maximin rule and/or in light of the dominance principle, and this is so despite our ignorance of the relevant probabilities concerning the existence/non-existence of convergent limits, the primitive improbability of inductive conclusions, and the improbability of the second-order claim that the inductive rule is correct. 


\section{References}

BonJour, L. (1986). A Reconsideration of the Problem of Induction. Philosophical Topics, 14, 93-124.

BonJour, L. (1992). Problems of Induction. In E. Sosa and J. Dancy (eds.), A Companion to Epistemology (pp. 391-395). Cambridge: Blackwell.

BonJour, L. (1998). In Defense of Pure Reason. Cambridge: Cambridge University Press.

Eberhardt, F. (2011). Reliability via Synthetic A Priori: Reichenbach's Doctoral Thesis on Probability. Synthese, 181, 125-136.

Feigl, H. (1963). De Principius Non Diputandum...? In M. Black (ed.), Philosophical Analysis: A Collection of Essays, (pp. 113-131). Engelwood Cliffs: Prentice Hall.

Galavotti, M. (2011). On Hans Reichenbach's Inductivism. Synthese, 181, 95-111.

Hájek, A. (1997). 'Mises Redux' - Redux. Fifteen Arguments against Finite Frequentism. Erkenntnis, 45, 209-227.

Hájek, A. (2009). Fifteen Arguments against Hypothetical Frequentism. Erkenntnis, 70, 211-235.

Kelly, K, (1991). Reichenbach, Induction and Discovery. Erkenntnis, 35, 123-149.

Kelly, K. (1996). The Logic of Reliable Inquiry. Oxford: Oxford University Press.

Reichenbach, H. (1949a). On the Justification of Induction. In H. Feigl and W. Sellars (eds.), Readings in Philosophical Analysis (pp. 324-329). New York: Appleton-Century-Crofts.

Reichenbach, H. (1932/1949b). The Logical Foundations of the Concept of Probability. In H. Feigl and W. Sellars (eds.), Readings in Philosophical Analysis (pp. 305-323). New York: Appleton-Century-Crofts. Originally published in 1932 as Die Logischen Grundlagen des Wahrscheinlichkeitsrechnung. Erkenntnis, 3, 410-425.

Reichenbach, H. (1949c). Theory of Probability. Berkeley: University of California Press.

Reichenbach, H. (1949d). A Letter to Bertrand Russell. In Reichenbach 1978, 405-411.

Reichenbach, H. (1978). Selected Writings, 1909-1953 (vol II). M. Reichenbach and W. C. Salmon (eds.). Boston: Dordrecht.

Resnik, M. (1987). Choices. Minneapolis: University of Minnesota Press.

Rosenkrantz, R. (1981). Foundations and Applications of Inductive Probability. Atascadero: Ridgeview Publishing.

Russell, B. (1911). On the Relations of Universals and Particulars. Proceedings of the Aristotelian Society, 12, 1-24. 
Russell, B. (1912). The Problems of Philosophy. New York: Oxford University Press.

Russell, B. (1948). Human Knowledge: Its Scope and Limits. New York: Clarion.

Salmon, W. (1963). On Vindicating Induction. In Sidney Luckenbach (ed.) Probabilities, Problems and Paradoxes (pp. 200-212). Encino: Dickenson.

Salmon, W. (1966). The Foundations of Scientific Inference. Oxford: Oxford University Press.

Skyrms, B. (1966). Choice and Chance: An Introduction to Inductive Logic. Belmont: Dickenson Publishing Co., Inc.

Teng, C. M. and H. Kyburg (2001). Uncertain Inference. Cambridge: Cambridge University Press.

Wald, A. (1939). Contributions to the Theory of Statistical Estimation and Testing Hypotheses. The Annals of Mathematics, 10, 299-326.

Wald, A. (1945). Statistical Decision Functions Which Minimize the Maximum Risk. The Annals of Mathematics, 46, 265-280.

Weatherford, R. (1982). Philosophical Foundations of Probability Theory. London: Routledge. 